\title{
Severe bradycardia and prolonged hypotension in ciguatera
}

Thomas Yan Keung $\underline{\text { Chan }}^{1}, \mathrm{MD}, \mathrm{PhD}$

ABSTRACT Ciguatera results when ciguatoxin-contaminated coral reef fish from tropical or subtropical waters are consumed. The clinical features that present in affected persons are mainly gastrointestinal, neurological, general, and much less commonly, cardiovascular. We report the case of a 50-year-old man who developed the characteristic combination of acute gastrointestinal and neurological symptoms after the consumption of an unidentified coral reef fish head. In addition to those symptoms, he developed dizziness, severe bradycardia (46 bpm) and prolonged hypotension, which required the administration of intravenous atropine and over three days of intravenous fluid replacement with dopamine infusion. Patients with ciguatera can develop severe bradycardia and prolonged hypotension. Physicians should recognise the possible cardiovascular complications of ciguatera and promptly initiate treatment with intravenous atropine, intravenous fluid replacement and inotropic therapy if such complications are observed.

Keywords: atropine, bradycardia, ciguatera, dopamine, hypotension

\section{INTRODUCTION}

Ciguatera results from the consumption of ciguatoxincontaminated coral reef fish from tropical or subtropical waters. ${ }^{(1,2)}$ These naturally-occurring ciguatoxins originate from the dinoflagellates species of the genus Gambierdiscus and bioaccumulate in food chains. The clinical features that present in affected persons are mainly gastrointestinal (e.g. abdominal pain, nausea, vomiting and diarrhoea), neurological (e.g. paraesthesia in the perioral areas and/or extremities, myalgia, muscle weakness, malaise and headache), general (e.g. pruritus and sweating), and much less commonly, cardiovascular (e.g. hypotension and bradycardia). ${ }^{(2,3)}$ The predominant features and the severity and duration of symptoms vary with geographical region, type and dose of ciguatoxin involved, as well as individual susceptibility. Neurological features predominate in the Indo-Pacific region, whereas gastrointestinal features predominate in the Caribbean. ${ }^{(4)}$ In the Indian Ocean region, additional symptoms of hallucinatory poisoning have been reported. ${ }^{(5)}$ Although cardiovascular features are rare $(0 \%-0.3 \%),{ }^{(2,3)}$ it is important for physicians to be aware that bradycardia and hypotension resulting from ciguatera can be severe. ${ }^{(1)}$ Severe cardiovascular symptoms will necessitate prompt treatment with intravenous atropine, fluid replacement and inotropic therapy.

\section{CASE REPORT}

A 50-year-old man with no past medical history presented to the hospital with epigastric pain, nausea, vomiting, diarrhoea, dizziness, malaise and paraesthesia of the extremities, three hours after consuming the head of a red coral reef fish bought from a market. The patient was not on any medications or supplements at the time of his admission. The species of the fish consumed could not be identified as the entire fish had been consumed. By the time the patient arrived at the hospital, which was 4.5 hours post ingestion, he had vomited twice and passed loose stools seven times. His pulse rate and blood pressure was 46 beats per minute (bpm) and 75/54 $\mathrm{mmHg}$, respectively. On examination, there was mild epigastric tenderness. Muscle power, light touch and pain sensation in the patient's four limbs were normal. Electrocardiogram (ECG) showed sinus bradycardia (heart rate 46 bpm) (Fig. 1). He was given $0.6 \mathrm{mg}$ atropine and $1,000 \mathrm{~mL} 0.9 \%$ saline intravenously at full rate, followed by $500 \mathrm{~mL}$ every six hours. Five minutes after the administration of atropine and saline, the patient's pulse rate increased from 51 bpmto $82 \mathrm{bpm}$ and his blood pressure increased from 93/51 mmHg to 120/72 $\mathrm{mmHg}$. After another 5-25 minutes had passed, his heart rate and blood pressure further increased to 100-120 bpm and 133-140/77-92 mmHg, respectively. 11.5-24 hours post ingestion, the patient experienced bradycardia (heart rate 53-57 bpm) and hypotension (blood pressure $75-86 / 34-46 \mathrm{mmHg}$ ) again, and was administered another $1,000 \mathrm{~mL} 0.9 \%$ saline intravenously at full rate. The patient was still hypotensive at 16-83 hours post ingestion, and thus required intravenous infusion of $0.9 \%$ saline $/ 5 \%$ dextrose (500 mL, $4-6$ hourly) and dopamine $(2.5 \mu \mathrm{g} / \mathrm{kg} / \mathrm{min})$ to prevent his systolic blood pressure from dropping below $100 \mathrm{mmHg}$.

On Day 1 of admission, intravenous metoclopramide (10 mg) and mannitol $(1 \mathrm{~g} / \mathrm{kg})$ over a duration of an hour, oral hyoscine butylbromide $(10 \mathrm{mg}$ ) and intramuscular tramadol $(75 \mathrm{mg}$ ) were prescribed. Plasma urea and creatinine concentrations on admission were $12.4 \mathrm{mmol} / \mathrm{L}$ (normal range $3.4-8.9 \mathrm{mmol} / \mathrm{L}$ ) and $79 \mu \mathrm{mol} / \mathrm{L}$ (normal range $62-106 \mu \mathrm{mol} / \mathrm{L}$ ), respectively, but decreased to $9.2 \mathrm{mmol} / \mathrm{L}$ and $71 \mu \mathrm{mol} / \mathrm{L}$, and $3.9 \mathrm{mmol} / \mathrm{L}$ and

Correspondence: Prof Thomas YK Chan, Professor and Consultant Physician, Division of Clinical Pharmacology, Department of Medicine and Therapeutics, Faculty of Medicine, The Chinese University of Hong Kong, Prince of Wales Hospital, Shatin, New Territories, Hong Kong, China. tykchan@cuhk.edu.hk 


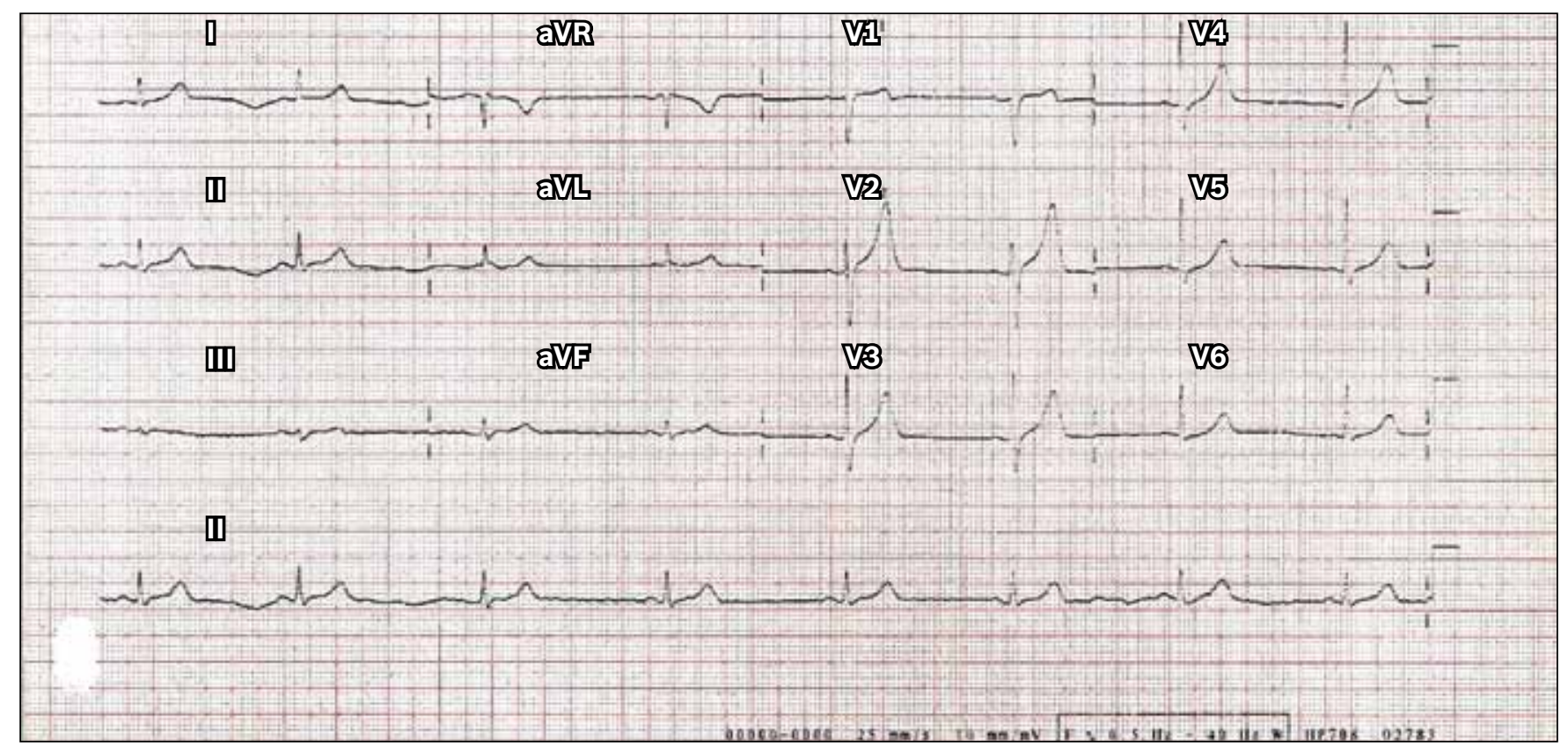

Fig. 1 ECG of the 50-year-old man with ciguatera shows sinus bradycardia (46 beats per minute).

$65 \mu \mathrm{mol} / \mathrm{L}$ after 9 and 30 hours of intravenous fluid therapy, respectively. Plasma electrolytes, cardiac troponin, transaminases, sensitive thyroid simulating hormone and complete blood counts were all normal. Vomiting and diarrhoea ceased after admission, and the patient ceased to experience mild dizziness and generalised paraesthesia by Day 2. The patient was discharged 89 hours post ingestion. His pulse rate and blood pressure on the day of discharge was 60-70 bpm and 100-104/46-64 mmHg, respectively. The patient's 50-year-old wife, who shared the fish head with him, experienced only a single day of diarrhoea, for which she had refused to seek medical treatment.

\section{DISCUSSION}

Ciguatera is diagnosed using the characteristic combination of gastrointestinal and neurological symptoms observed in patients who have consumed large, predatory coral reef fish, which generally weigh more than 2 kg. ${ }^{(2)}$ In Hong Kong, cases of ciguatera had been related to the consumption of live coral reef fish imported from areas such as Nan Sha Islands and South Pacific. According to past reports of ciguatera, fish species that are more likely to contain ciguatoxins include moray eel, potato grouper, speckled blue grouper, tiger grouper, high fin grouper, humphead wrasse, areolated coral grouper, blacksaddled coral grouper, lyretail, blackfin red snapper, flowery grouper and leopard coral grouper. ${ }^{(6)}$ Most (80\%) people develop symptoms within 12 hours of consuming the fish. ${ }^{(2)}$ Although gastrointestinal symptoms usually subside within days, neurological symptoms may last for weeks or longer. ${ }^{(2)}$ Severe bradycardia and hypotension are among the more serious complications of ciguatera reported in Hong Kong, but fortunately, such complications are rare $(0 \%-0.3 \%) .^{(2,3)}$

The present case demonstrates that hypotension due to ciguatera can be prolonged, lasting over three days. Dehydration and hypovolaemia as a result of vomiting and diarrhoea should be corrected using adequate intravenous fluid replacement. As our patient's plasma urea concentration $(9.2 \mathrm{mmol} / \mathrm{L})$ was almost normal after nine hours of intravenous fluid therapy, dehydration and hypovolaemia as a cause of hypotension was excluded. The reversal of bradycardia by intravenous atropine, the marked pressor response to low-dose dopamine or intravenous noradrenaline infusion, and the low plasma catecholamine levels suggest that hypotension observed in ciguatera is the result of both parasympathetic excess and sympathetic failure. ${ }^{(1,7)}$

An effective antidote is not available, and treatment for ciguatera is essentially supportive. Intravenous mannitol infusion has been suggested as treatment after case studies and uncontrolled observations reported marked improvements in the symptoms of some patients. ${ }^{(8,9)}$ However, a double-blind, randomised controlled study of 50 patients with ciguatera in Rarotonga, Cook Islands, revealed that normal saline was as effective as mannitol in relieving the symptoms of ciguatera at 24 hours. ${ }^{(10)}$ In the study, $500 \mathrm{~mL}$ intravenous normal saline infusion and $500 \mathrm{~mL}$ intravenous mannitol (20\% solution) were given to $76 \%$ and $92 \%$ of patients, respectively, within $12-24$ hours of symptoms onset. More than $70 \%$ of the patients in the study were followed up for at least one week and the prevalence of asymptomatic patients at one week was similar (28\%) in each group. ${ }^{(10)}$

Severe bradycardia and prolonged hypotension can occur in ciguatera. Physicians should recognise the potential cardiovascular complications of ciguatera and promptly initiate treatment with intravenous atropine, intravenous fluid replacement and inotropic therapy if such complications are observed. To prevent ciguatera, the public should avoid eating large coral reef fish - especially the head, viscera, skin and roe, 
which usually contain a higher concentration of ciguatoxins. ${ }^{(2,6)}$ The Centre for Health Protection of the Department of Health in Hong Kong has reminded the public to be extra cautious when consuming coral reef fish as the fish may contain ciguatoxins, which can cause food poisoning.

\section{REFERENCES}

1. Chan TYK, Wang AYM. Life-threatening bradycardia and hypotension in a patient with ciguatera fish poisoning. Trans $\mathrm{R}$ Soc Trop Med Hyg 1993; 87:71.

2. Choi SMY, Wong MMH. Epidemiology of ciguatera poisoning in Hong Kong. Public Health Epidemiol Report 1994; 3:12-4.

3. Au A. Ciguatera fish poisoning on the rise - a review of cases (January 2004-May 2005). Communicable Diseases Watch 2005; 13:49-50.

4. Isbister GK, Kiernan MC. Neurotoxic marine poisoning. Lancet Neurol 2005; 4:219-28.
5. Quod JP, Turquet J. Ciguatera in Réunion Island (SW Indian Ocean): epidemiology and clinical patterns. Toxicon 1996; 34:779-85.

6. Seasonal food safety tips - prevention of ciguatera fish poisoning start with choosing coral reef fish carefully. Centre for Food Safety [online]. Available at: http://www.cfs.gov.hk/english/whatsnew/ whatsnew_fsf/whatsnew_fsf_poison_fish.html. Accessed December 30, 2006.

7. Geller RJ, Benowitz NL. Orthostatic hypotension in ciguatera fish poisoning. Arch Intern Med 1992; 152:2131-3.

8. Palafox NA, Jain LG, Pinano AZ, et al. Successful treatment of ciguatera fish poisoning with intravenous mannitol. JAMA 1988; 259:2740-2.

9. Pearn JH, Lewis RJ, Ruff T, et al. Ciguatera and mannitol: experience with a new treatment regimen. Med J Aust 1989; 151:77-80.

10. Schnorf H, Taurarii M, Cundy T. Ciguatera fish poisoning: a doubleblind randomized trial of mannitol therapy. Neurology 2002; 58:873-80. 